\title{
PAPER \\ A Two-Stage Point Pattern Matching Algorithm Using Ellipse Fitting and Dual Hilbert Scans
}

\section{Li TIAN $^{\dagger a)}$, Student Member and Sei-ichiro KAMATA ${ }^{\dagger b)}$, Member}

SUMMARY Point Pattern Matching (PPM) is an essential problem in many image analysis and computer vision tasks. This paper presents a two-stage algorithm for PPM problem using ellipse fitting and dual Hilbert scans. In the first matching stage, transformation parameters are coarsely estimated by using four node points of ellipses which are fitted by Weighted Least Square Fitting (WLSF). Then, Hilbert scans are used in two aspects of the second matching stage: it is applied to the similarity measure and it is also used for search space reduction. The similarity measure named Hilbert Scanning Distance (HSD) can be computed fast by converting the 2-D coordinates of 2-D points into 1-D space information using Hilbert scan. On the other hand, the N-D search space can be converted to a 1-D search space sequence by N-D Hilbert Scan and an efficient search strategy is proposed on the 1-D search space sequence. In the experiments, we use both simulated point set data and real fingerprint images to evaluate the performance of our algorithm, and our algorithm gives satisfying results both in accuracy and efficiency.

key words: point pattern matching, ellipse fitting, Hilbert scan, transformation parameter estimation, search space reduction

\section{Introduction}

Point Pattern Matching (PPM) is an important problem found in a variety of applications such as image registration, object recognition and stereo vision. PPM algorithm always matches two point patterns by finding an optimal transformation where a similarity (dissimilarity) measure of the two point patterns is minimized (maximized). PPM can be divided into complete and incomplete cases based on if there exists a one to one mapping between the two point sets to be matched, and it can also be divided into labelled and unlabelled cases based on if additional information such as intensity, color and gradient other than 2-D image coordinates are used [1]. Hence, PPM problems can be classified into four different types as shown in Fig. 1. Of all these four types, type 4 is the most difficult one and also is the most suitable one for describing many real-world image and vision applications. In this study, we concentrate on this type under the affine transformation. Two factors are important in this type of PPM problem: the similarity measure and the search strategy.

Many approaches have been developed for the two factors mentioned above. Two well-known similarity measures

Manuscript received December 18, 2007.

Manuscript revised June 24, 2008.

†The authors are with the Graduate School of Information, Production and Systems, Waseda University, Kitakyushu-shi, 8080135 Japan.

a) E-mail: tianli@ruri.waseda.jp

b)E-mail: kam@waseda.jp

DOI: 10.1093/ietisy/e91-d.10.2477

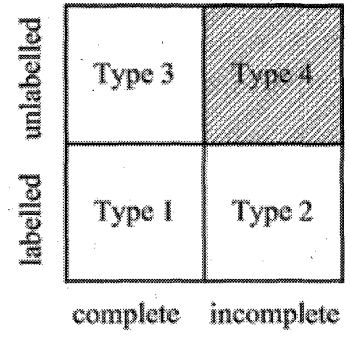

Fig. 1 Types of point pattern matching problem.

called Hausdorff distance (HD) and partial Hausdorff distance (PHD) have been widely used for PPM [2]. Modified Hausdorff distance (MHD) [3] is an extension of them. A heuristic dissimilarity measure is also proposed in [4]. On the other hand, many search strategies including clustering methods [5], [6], parameter decomposition methods [7], [8], relaxation methods [9], [10], bounded alignment [11], spectral graph analysis [12], genetic algorithms [13], simulated annealing [14] and particle swarm optimization [1] also have been proposed for PPM problems. Among them, only a few of them are suitable for type 4 and many of them have the assumption that two point patterns are already coarsely aligned, which is not feasible in real-world applications.

In this study, we propose a two-stage point pattern matching algorithm using ellipse fitting and Dual Hilbert scans. In the first (or coarse) matching stage, a four-point estimation method based on axis node points of ellipses obtained by a fitting method named Weighted Least Square Fitting (WLSF) is proposed for PPM. In the second (or fine) matching stage, Hilbert scans are used twice in the matching process: first it is applied to the similarity measure named Hilbert Scanning Distance (HSD) which can be computed fast by converting the 2-D coordinates of 2-D points into 1D space information using Hilbert scan; and then it is used to convert the N-D search space to a 1-D search space sequence and a fast search strategy, which is similar to gradient descent, is proposed on the 1-D search space sequence. In the experiments, we evaluate our algorithm using both simulated data and real fingerprint images for PPM problem. In the first matching stage, WLSF shows more robustness to noise and outlier points than Direct Least Square Fitting (DLSF) in ellipse fitting and the search space can be reduced to a small range. The whole algorithm also gives satisfying results both in accuracy and efficiency for PPM problem.

This study gives four major contributions to ellipse fit- 
ting method and PPM problem:

1. WLSF provides a more robust fitting method for noise and outlier points than DLSF in PPM problem.

2. The coarse transformation parameter estimation using four axis node points of ellipses is stable and can reduce the search space into a small range to save searching time much in PPM problem.

3. A search strategy based on 1-D Hilbert scanned search space is proposed and it has flexibility between accuracy and efficiency.

4. Making use of dual Hilbert scans for PPM is effective and it is suitable for hardware.

The remainder of this paper is organized as follows. First, we discuss our previous work including 3-D Hilbert scan and HSD in Sect. 2; then, details about the proposed WLSF and the first matching stage is given in Sect. 3; Section 4 is about the second matching stage using dual Hilbert scans; Section 5 presents experimental results using both simulated data and real fingerprint images and gives some discussions. Conclusions and future work are given in the last section.

\section{Previous Work}

In this section, we will introduce two important techniques related to the proposed algorithm: 3-D Hilbert Scan and HSD. Hilbert Scan is based on the Hilbert spacefilling curves. Space-filling curves are curves whose ranges contain the entire 2-dimensional unit square (or the 3dimensional unit cube, or $n$-dimensional hypercube). They can convert N-D space to 1-D space by one-to-one mapping. In 1890 , Peano discovered a densely self-intersecting curve named Hilbert curve which passed through every point of the unit square which is different from Lebesgue curve and Zig-zag curve. Hilbert curve has been widely applied in image processing tasks such as image compression, image clustering, pattern recognition [15]-[18] since it has the property that it preserves point neighborhoods as much as possible. Our matching algorithm is inspired and based on the mentioned work.

\subsection{3 -D Hilbert Scan}

The Hilbert curve is a locus of points in N-D space. For its special attributes, it becomes an analytical solution of a space-filling curve. In 1891, Hilbert made a curve having the space-filling property in 2-D space. The generation of a 3-D Hilbert curve is described in [19]. It demonstrated that the sub-cubes can be arranged so that the inclusion relationships are preserved, that is, if a cube corresponds to an interval, then its sub-cubes correspond to subintervals of that interval.

A one-to-one mapping whose domain is a set of $P$ nonnegative integers $(I)$ and whose image is a set of $P$ points $(Q)$, is called a scan. If a point $\boldsymbol{a}(\boldsymbol{a} \in Q)$ is the image of an integer $t(t \in I)$ under $f$, i.e. $t \stackrel{f}{\longrightarrow} \boldsymbol{a}$, then $\boldsymbol{a}$ is denoted by $f(t)$. In particular, $f(0)$ is called the entry, and $f(P-1)$

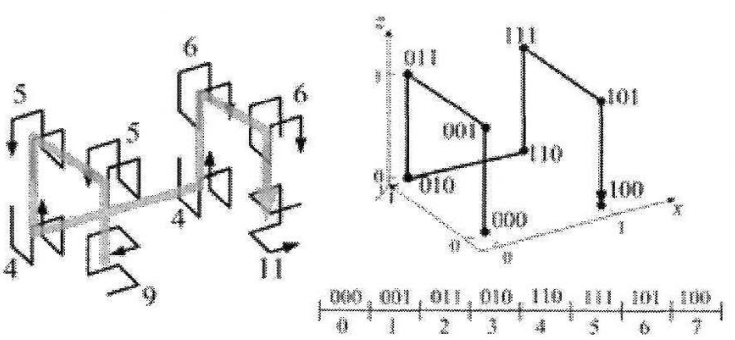

Fig. 2 Address and curve types of Hilbert scan.

is called exit of the set of points. Here, we assume that a 3-D Euclidean space with finite granularity. We use the $m t h$ order approximation of a 3-D Hilbert curve $(m \geq 1)$, which maps an integer set $\left[0,2^{3 m}-1\right]$ into a 3-D integer space $\left[0,2^{m}-1\right]^{3}$. Let $S_{3}^{m}$ denote a three-dimensional Hilbert curve with the size of $2^{m} \times 2^{m} \times 2^{m}$ mapping the integer set $\left[0,2^{3 m}-1\right]$ into $\left[0,2^{m}-1\right]^{3}$, where $m(m \geq 1)$ is the rank corresponding to the size of the cube. In the $S_{3}^{m}$ case, the set $I$ and $Q$ are $\left[0,2^{3 m}-1\right]$ and $\left[0,2^{m}-1\right]^{3}$, respectively; the entry and exit are $f(0)$ and $f\left(2^{3 m}-1\right)$, respectively. Figure 2 shows the address and curve types for constructing 3-D Hilbert scan. In the figure, the binary and decimal sequence numbers express the address alignment and scanning patterns respectively. More details about 3-D Hilbert Scan for arbitrarily-sized arrays can be found in work [20].

There are two important features of Hilbert scan:

1. Extracting a group of neighboring pixels having similar intensities is easier by using Hilbert scan than other scan techniques such as raster scan, row-prime scan and Morton scan.

2. A Hilbert scan preserves the coherence in a 3-D space. If the Euclidean distance between two points in the 3 $\mathrm{D}$ space is small, then the scanning length between the same pair of points in the 1-D sequence is also small.

The two features are quite useful for our algorithm. The spacial properties and one to one mapping of Hilbert scan benefit the proposed similarity measure and search strategy in this study.

\subsection{Hilbert Scanning Distance}

HSD is proposed as a similarity measure and utilized for matching point sets. We don't discuss the details that how to compute HSD [21] in this study. Here, we only summarize the definition and brief computing process of HSD.

Assume that we are given two finite point sets $A=$ $\left\{a_{1}, \ldots, a_{I}\right\}$ and $B=\left\{b_{1}, \ldots b_{J}\right\}$ such that each point $a \in A$ and $b \in B$ has integer coordinates in the 2-D space. We firstly use Hilbert scanning to convert them to new sets $S=\left\{s_{1}, \ldots, s_{I}\right\}$ and $T=\left\{t_{1}, \ldots t_{J}\right\}$ in the $1-\mathrm{D}$ sequence, respectively. Then, the directed $\mathrm{HSD}$ from $A$ to $B h_{h s d}(A, B)$ is computed by

$$
h_{h s d}(A, B)=\frac{1}{I} \sum_{i=1}^{I} \rho\left(\min _{j}\left\|s_{i}-t_{j}\right\|\right)
$$


where $\|\cdot\|$ is the Euclidean norm distance in the 1-D space and function $\rho$ is defined as:

$$
\rho(x)= \begin{cases}x & (x \leq \tau) \\ \tau & (x>\tau)\end{cases}
$$

where $\rho$ is called threshold elimination function and $\tau$ is a threshold predefined. We also can obtain the directed HSD from $B$ to $A h_{h s d}(B, A)$ similarly and HSD is defined by

$$
H_{h s d}(A, B)=\max \left(h_{h s d}(A, B), h_{h s d}(B, A)\right)
$$

If two binary images $A$ and $B$ including feature points have been given, we first construct a new image $C$ by combining them under a certain transformation. Note that there must be 3 types of feature point in the combined image $C$ : feature point from $A$, feature point from $B$ and overlapped feature point from both $A$ and $B$. In order to distinguish the 3 types of point, we give values 1,2 and 3 to them, respectively. Then, the process of computing $h_{h s d}(A, B)$ can be summarized as the following steps:

Step1 Using a prepared lookup table to convert the combined image to a 1-D sequence $C^{\prime}$;

Step2 For any feature point $c_{i}^{\prime}$ in $C^{\prime}$ : if (value of $c_{i}^{\prime}$ ) $=2$, next feature point; else if (value of $c_{i}^{\prime}$ ) $=1$, find the nearest feature point $c_{i}^{\prime \prime}$ whose value is 2 or 3 , and the distance $d_{i}=\left\|c_{i}^{\prime}-c_{i}^{\prime \prime}\right\|$; else (value of $c_{i}^{\prime}$ ) $=3, d_{i}=0$;

Step3 If $d_{i}>\tau$, then $d_{i}=\tau$. This step can also be done by specifying the search range in Step 2 more efficiently;

Step4 Repeat Step2 and Step3 until all the feature points have been processed;

Step5 Obtain $h_{h s d}(A, B)$ by calculating the mean value of all $d_{i}$;

Step6 Stop.

\section{First Matching Stage}

Fitting geometrical models to image data is important in image processing and computer vision fields since it can reduce and simplify the data for high level image processing task. Among various geometrical models, ellipse is the most commonly used one. Ellipse fitting methods can be categorized into two types: clustering methods such as Houghbased methods [22]-[25] and least square fitting methods. In this study, we focus on the latter type. Most of the least square fitting methods concentrate on finding the parameters to minimize some distance measure between the ellipse and the data points. Different constraints [26]-[30] applied to the parameters are used to obtain the solution. Many refinement methods are also developed to improve the accuracy of fitting results [31]-[33]. Among all these methods, DLSF is reported as the most robust one to noise [30]. This section introduces a modified fitting method WLSF and discuss how to estimate the transformation parameters using fitted ellipses in the first matching stage.

\subsection{Ellipse Fitting}

A general quadratic curve can be presented as an implicit second order polynomial:

$$
F(\mathbf{a} ; \mathbf{x})=a x^{2}+b x y+c y^{2}+d x+f y+g=0
$$

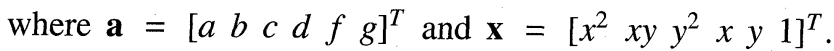
In DLSF [30], $F\left(\mathbf{a} ; \mathbf{x}_{i}\right)$ is called the algebraic distance of a point $(x, y)$ to the quadratic curve $F(\mathbf{a} ; \mathbf{x})=0$. In our method, we give a weight $w_{i}$ to each algebraic distance of a point $(x, y)$ and called it as weighted algebraic distance:

$$
G\left(\mathbf{a} ; \mathbf{x}_{i}\right)=w_{i} F\left(\mathbf{a} ; \mathbf{x}_{i}\right) .
$$

We will discuss how to compute the weight later. The fitting of the quadratic curve can be obtained by minimizing the sum of squared weighted algebraic distances

$$
Q_{A}(\mathbf{a})=\sum_{i=1}^{N} G\left(\mathbf{x}_{i}\right)^{2}
$$

of $N$ data points $\mathbf{x}_{i}$. The minimization can be solved by using deficient generalized eigenvalue system as follows:

$$
\mathbf{Q}^{T} \mathbf{Q} \mathbf{a}=\lambda \mathbf{C a}
$$

where $\mathbf{Q}=\left[\mathbf{x}_{1} \mathbf{x}_{2} \cdots \mathbf{x}_{n}\right]$ is the design matrix and $\mathbf{C}$ is the constraint. We use the same constraint $4 a c-b^{2}=1$ as in DLSF. Now, the constrained ellipse problem can be solved by minimizing the Eq. (6) subject to this constraint. For more details about how to solve the system, please refer to [26], [30].

Now, we are beginning to discuss how to compute the weight for each point in a point set. Given a point set $P=$ $\left\{\mathbf{p}_{i}=\left(x_{i}, y_{i}\right) \mid i=1,2, \ldots, n\right\}$ in $\mathbb{R}^{2}$. First, we compute the central point ${ }^{\dagger} \mathbf{p}_{c}=\left(x_{c}, y_{c}\right)$ as

$$
x_{c}=\frac{1}{N} \sum_{i=1}^{N} x_{i}, y_{c}=\frac{1}{N} \sum_{i=1}^{N} y_{i} .
$$

Then, for each point in this point set, compute the Euclidean distance $d_{i}$ from it to the central point. Finally, the weight of a point in the point set is computed as

$$
w_{i}=\left\{\begin{array}{lll}
1 & \text { if } & d_{i} \leq r_{1} \\
1-\left(d_{i}-r_{1}\right) /\left(r_{2}-r_{1}\right) & \text { if } & r_{1}<d_{i}<r_{2} \\
0 & \text { if } & d_{i} \geq r_{2}
\end{array}\right.
$$

where $r_{1}$ and $r_{2}(r 1<r 2)$ are two thresholds predefined.

Figure 3 gives an illustration that how to compute the weights: if a point is close to the central point as in region $R_{1}$, the weight is 1 , that means it gives a full algebraic distance to the Eq. (6); if a point is a little far from the central point as in region $R_{2}$, its contribution to Eq. (6) is weakened

${ }^{\dagger}$ In some matching applications, feature point also can be used instead of the central point, i.e, core point are used as the central point in fingerprint images. 


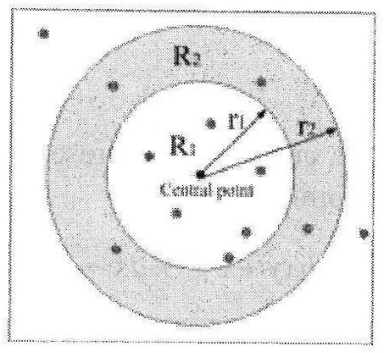

Fig.3 Illustration of how to compute the weights.

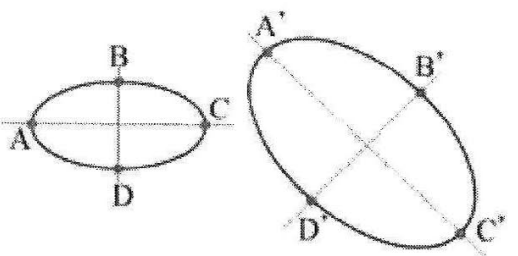

Fig. 4 Four axis node points of ellipses.

based on its distance to the central point; if a point is far away from the central point, it will be eliminated. The proposed WLSF method is similar to DLSF, particularly, if the parameter $r_{1}$ is large enough, WLSF becomes the same form as DLSF. However, the weights used in WLSF can help the fitting method to improve its robustness to noise and outlier points in many real matching problem such as fingerprint matching.

\subsection{Parameter Estimation}

In PPM problem, if the transformation space is large, it will takes a long time to find the optimal transformation position. Hence, techniques for reducing the search space to a local space around the optimal transformation position are highly desired. In our study, we propose a simple but powerful method to estimate the transformation parameters using the ellipses obtained by WLSF.

Generally, linear conformal, affine and projective transformation can be obtained by giving a pair of two, three and four corresponding points, respectively [34]. Here, we discuss the four-point case and the two and three-point cases can be included in the four-point case. Given a fitted ellipse, four node points of major and minor axes of the ellipse are selected to compute the projective transformation as shown in Fig. 4. Notice that there are four corresponding candidates (two in linear conformal and affine transformation): $\left(A \leftrightarrow A^{\prime}, B \leftrightarrow B^{\prime}, C \leftrightarrow C^{\prime}, D \leftrightarrow D^{\prime}\right),\left(A \leftrightarrow C^{\prime}, B \leftrightarrow\right.$ $\left.D^{\prime}, C \leftrightarrow A^{\prime}, D \leftrightarrow B^{\prime}\right),\left(A \leftrightarrow A^{\prime}, B \leftrightarrow D^{\prime}, C \leftrightarrow C^{\prime}, D \leftrightarrow B^{\prime}\right)$, and $\left(A \leftrightarrow C^{\prime}, B \leftrightarrow B^{\prime}, C \leftrightarrow A^{\prime}, D \leftrightarrow D^{\prime}\right)$. The false three can be easily rejected by comparing some similarity measure such as correlation of the image or the Hausdorff distance [2].

The projective transformation $\mathbf{x}^{\prime}=\mathbf{H x}$ can be expressed in a matrix form

$$
\left(\begin{array}{l}
x_{1}^{\prime} \\
x_{2}^{\prime} \\
x_{3}^{\prime}
\end{array}\right)=\left[\begin{array}{lll}
h_{11} & h_{12} & h_{13} \\
h_{21} & h_{22} & h_{23} \\
h_{31} & h_{32} & h_{33}
\end{array}\right]\left(\begin{array}{l}
x_{1} \\
x_{2} \\
x_{3}
\end{array}\right)
$$

where $\mathbf{H}$ is called the homogeneous matrix and $\left(x_{1}, x_{2}\right) \leftrightarrow$ $\left(x_{1}^{\prime}, x_{2}^{\prime}\right)$ is the point correspondence in Cartesian coordinates. Following the work in [35], we can generate two linear equations for the elements of $\mathbf{H}$

$$
\begin{aligned}
& x_{1}^{\prime}\left(h_{31} x_{1}+h_{32} x_{2}+h_{33}\right)=h_{11} x_{1}+h_{12} x_{2}+h_{13} \\
& x_{2}^{\prime}\left(h_{31} x_{1}+h_{32} x_{2}+h_{33}\right)=h_{21} x_{1}+h_{22} x_{2}+h_{23} .
\end{aligned}
$$

If we have $n \geq 4$ points (no three points collinear), then the homogeneous matrix $H$ can be determined uniquely. After obtaining the homogeneous matrix, the transformation parameter can be easily obtained and a local search space around it can be used to search the optimal transformation in the next stage.

\section{Second Matching Stage}

In this section, we will explain how to obtain the optimal transformation parameters by using Hilbert scanning technique. Here, we take the 3-D Hilbert scan as the example. So, horizontal translation $\Delta x$, vertical translation $\Delta y$ and rotation $\theta$ are considered as the transformations. If the number transformation parameters is larger than 3 , it can also be solved by using N-D Hilbert scan.

Thus, the second matching stage can be listed as following steps:

1. Construct the 3-D parameter space coordinates $(\Delta x, \Delta y, \theta)$.

2. Sample transformation space to discrete points.

3. Use 3-D Hilbert scan to convert the 3-D parameter space into 1-D space.

4. Find the optimal parameters in the search space using HSD as the similarity measure.

We use Hilbert scan to convert the 3-D space into 1D sequence as $H=\left\{H_{1}, H_{2}, \ldots, H_{L}\right\}$ where $L$ is the total number of the points in the 3-D parameter space. Each point in the 1-D sequence corresponds to a transformation and has an HSD value. We should find a point whose distance is the smallest. Of course, when the number of the points becomes large, it takes much time to do the full search. Here we will show how to search more efficiently.

Some notions used in the search algorithm are as follows:

$C_{i}$ : the $i$ th candidate point in current candidate set;

$S=\left\{C_{i} \mid i=1,2,3 \ldots, N\right\}$ : current candidate set including $N$ candidates;

$M_{i}:$ the middle point of the left or right adjacent intervals of $C_{i}$;

$C_{m i n}$ : the candidate point with the smallest HSD value in current candidate set;

$G_{\min }$ : the candidate point with the smallest HSD value in whole search procedure; 
$H S D(x):$ the directed HSD value of a candidate point $x$ $F(x)$ : a selection function for keeping the candidate $x$ to the next search stage. follows:

The algorithm which is similar to gradient descent is as

Step 1 Initialize $G_{\min }$ with a large number and equally divide the whole 1-D sequence into $k$ parts using $k+1$ initial points with interval $\Delta d=\Delta d_{0}$.

Step 2 Form $S$ with the $k+1$ initial points.

Step 3 For $C_{i}$, compare its two adjacent points $C_{i}+\Delta d$ and $C_{i}-\Delta d$. If $H S D\left(C_{i}+\Delta d\right) \leq H S D\left(C_{i}-\Delta d\right), M_{i}=$ $C_{i}+\Delta d / 2 ;$ otherwise, $M_{i}=C_{i}-\Delta d / 2$;

Step 4 Compare $M_{i}$ to $C_{i}$, if $H S D\left(M_{i}\right) \leq H S D\left(C_{i}\right)$, replace $C_{i}$ with $M_{i}$;

Step 5 Repeat Steps 3 and 4 until all candidates are processed.

Step 6 Find $C_{\min }$ from $S$, if $C_{\min } \leq G_{\min }, G_{\min }=C_{\min }$;

Step 7 Update $S$ using the selection function $F(x)$;

Step $8 \Delta d=\Delta d / 2$. If $\Delta d \geq 1$, go to step 3); otherwise, stop and go to next step;

Step 9 Let point with $G_{\min }$ be the optimal solution.

In Step 7, the selection function is defined as

$$
F(x)=\frac{H S D(x)-H S D\left(G_{\min }\right)}{H S D(x)}-t, 0 \leq t \leq 1
$$

where $t$ is a threshold to control the selection. When $t$ is smaller, fewer candidates will be selected. An extreme case is that when $t$ is 0 , only one candidate is selected in each stage. Thus, $t$ gives flexibility between speed and accuracy in the search.

\section{Experimental Results and Discussions}

In this section, we present the experimental results using our algorithm and analyze the computational complexity. The first experiment is designed for comparing the performance DLSF and WLSF in the first matching stage using both simulated point data and real fingerprint images. The other experiment illustrates the performance of the whole algorithm in real fingerprint matching problem. We also analyze the computational complexity of our algorithm at last.

\subsection{Experiment One}

We first evaluate the estimated results by using simulated point data. The first column of Fig. 5 shows a point set consisting 250 points generated randomly. We set up two scenarios to generate testing patterns to be matched: a point set by deleting $20 \%$ points randomly and a point set by adding $20 \%$ points randomly are shown in the second and third columns of Fig. 5, respectively. The fitting results by DLSF and WLSF are painted as the ellipses in Fig. 5. The $r_{1}$ and $r_{2}$ are set to 100 and 200, respectively. Then, these ellipses are used to compute the transformation parameters following the proposed method using four axis node points.

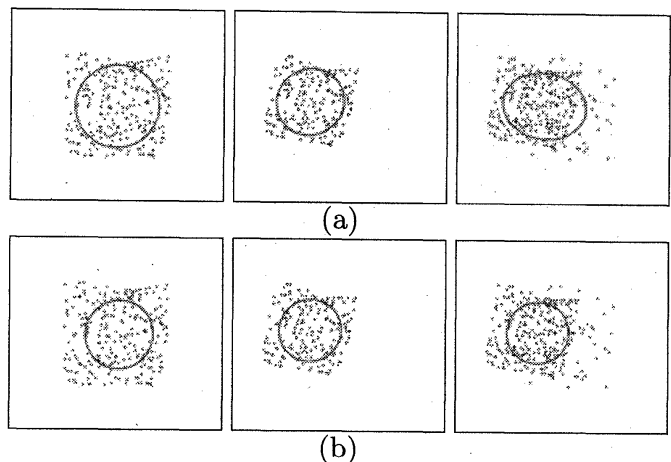

Fig. 5 Fitting results of DLSF and WLSF on simulated data. (a) Results obtained by DLSF. (b) Results obtained by WLSF.

Table 1 The estimation parameters on simulated data.

\begin{tabular}{|c|c|c|}
\hline \multirow{2}{*}{} & \multicolumn{2}{|c|}{$\left(\mathrm{s}, \theta^{\circ}\right)$} \\
\cline { 2 - 3 } & DLSF & WLSF \\
\hline Delete points & $(1.0,-14.1)$ & $(1.0,-1.3)$ \\
\hline Add points & $(1.0,34.2)$ & $(1.0,7.6)$ \\
\hline Ave. error & $(0,24.2)$ & $(0,4.5)$ \\
\hline
\end{tabular}

Table 1 shows the results of the estimated transformation parameters. We can observe that the estimated parameters by WLSF is much closer to the optimal ones than those by DLSF, and the search space can be reduced to a small space using the estimated parameters.

Fingerprint matching is an important application and most automatic fingerprint identification systems match fingerprint images based on the minutia points [36] as a PPM problem. Figures $6(\mathrm{a})-(\mathrm{c})$ show three fingerprint images taken from the same finger at different time with all minutia points extracted. As mentioned previously, we use the upper core point in WLSF as the central point in this experiment. The alignment results by using the estimated transformation parameters from DLSF and WLSF are shown in Fig. 6 (d)(g). From these figures, it is seen that the results obtained by WLSF are much better than by DLSF, where the core points are much closer or nearly overlapped in alignment results.

\subsection{Experiment Two}

In this experiment, we use the proposed method for fingerprint matching problem. After minutia points are extracted, we treat the fingerprint matching as PPM problem. Because the fingerprint matching is strongly affected by the minutia extraction and non-rigid deformation (however, we only consider the translation and rotation in this experiment) which often occurs in fingerprint images, the purpose of this experiment is not to show our method is the best method for fingerprint matching, but to show the effectiveness of our method for PPM problem. Suppose we have obtained the best parameters $(\Delta x, \Delta y, \theta)$ using the method we proposed, we can align the two minutia point patterns. Let $P\left(m_{i}, m_{j}\right)$ be an indicator function that return 1 if the minutiae $m_{i}$ and $m_{j}$ are matched by comparing their distance difference $D\left(m_{i}, m_{j}\right)$ and direction difference $R\left(m_{i}, m_{j}\right)$ : 

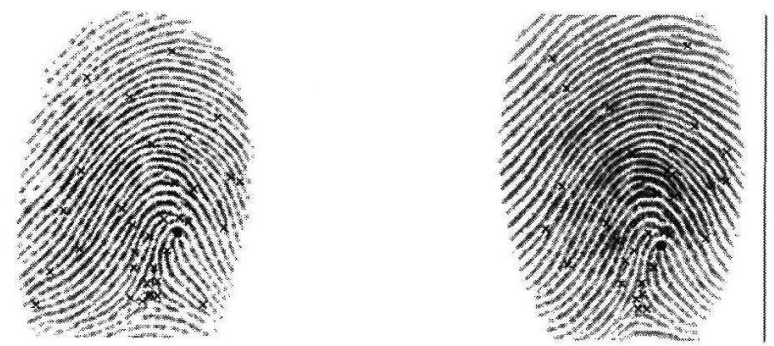

(b)
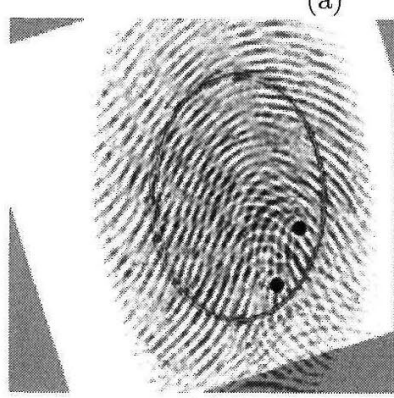

(d)

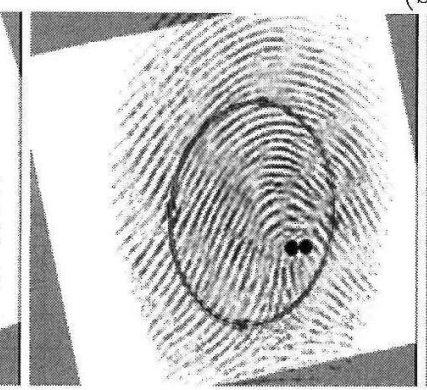

(e)

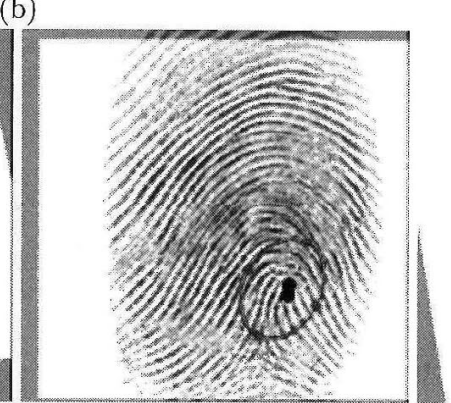

(f)

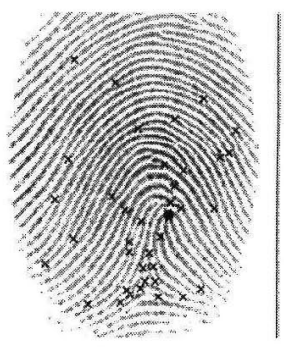

(c)

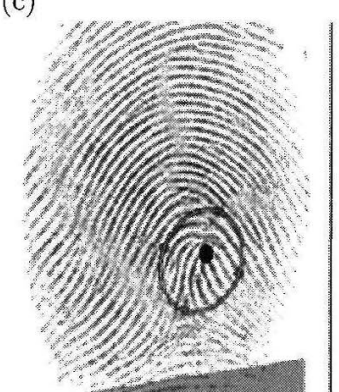

(g)

Fig. 6 Alignment results of fingerprint images (cross means minutiae point and black circle means core point). (a) Fingerprint A. (b) Fingerprint B. (c) Fingerprint C. (d) A to B by DLSF. (e) A to C by DLSF. (f) A to B by WLSF. (g) A to C by WLSF.

$$
P\left(m_{i}, m_{j}\right)=\left\{\begin{array}{ll}
1 & D\left(m_{i}, m_{j}\right) \leq d_{0} \& R\left(m_{i}, m_{j}\right) \leq \theta_{0} \\
0 & \text { otherwise }
\end{array} .\right.
$$

When the function $P$ gets value 1, we define these two minutia points as matched pair. We also define the matching score $Q$ to quantize the effect of the matching procedure:

$$
Q=\frac{\# \text { matchedpair }}{\max \{M, N\}}
$$

where \#matched pair is the number of the matched pairs, $M$ and $N$ are the numbers of the minutia points in template and input images, respectively. So, the fingerprint matching problem can be simplified as if the $Q \geq T_{0}$, then we definc the template and input images as matched.

We take a part of FVC2002 database [37] as the test database in our experiment. We use 800 fingerprint images (100 fingers and 8 impressions for each finger) in FVC2002 DB1_a. Each sample in the subset of a finger is matched against the remaining samples of the same finger to compute the False Rejection Rate (FRR), also referred as False Non Match Rate (FNMR). The first sample of each finger in the subset is matched against the first sample of the remaining fingers to compute the False Acceptance Rate (FAR), also referred as False Match Rate (FMR). For more details about the evaluation, please refer to [37]. The computational time of each matching is about $0.5 \mathrm{~s}$ by using $\mathrm{c}++$ on a PC with $2.0 \mathrm{Ghz}$ CPU and $1 \mathrm{G}$ memories. The Equal Error Rate (EER) of the experiment is about $14 \%$ as shown in Fig. 7.

Some parameters used in our algorithm are listed as follows:

- The search ranges of $x, y$ translations and rotation are

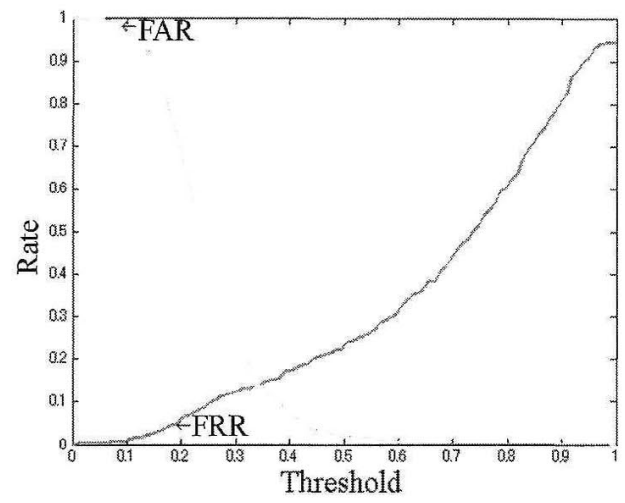

Fig. 7 The EER result of the fingerprint matching.

$[-5,6],[-5,6],[-p i * 5 / 48, p i / 8]$ with a pre-alignment of the template and the input images using the estimated transformation parameters.

- All fingerprints are cropped to $128 \times 128$ pixels, which is also the size of 2-D Hilbert scan for computing HSD value, centered around the core point (if there is no core point, we define the position of a dummy core point as the mean value of minutia point coordinates).

- The 3-D parameter search space are sampled to $12 \times$ $12 \times 12$ points, which is also the size of 3 -D Hilbert scan.

- The threshold value $\tau$ for computing HSD value is 50 .

- The number of initial points in 1-D sequence is 9 .

- The threshold value $d_{0}$ and $\theta_{0}$ for matching point pairs are 3 and 0.1 , respectively.

- The threshold value $t$ for selecting candidate is 0.5 . 
The EER of our proposed method may not be comparable to other competitors in the fingerprint authentication, but considering the purpose of this experiment indicated above and we only use the minutia point information, it shows the effectiveness of our method for computing the transformation parameters in PPM problem.

\subsection{Complexity Analysis}

The efficiency of our algorithm includes two aspects: one is from computing the similarity measure HSD and the other is from the search strategy.

Hausdorff distance is a commonly used similarity measure for point pattern matching [2]. If $M$ and $N$ are the numbers of minutiae points in the template and the input images and no efficient data structure such as K-D tree for nearest neighbor searching is used, the number of points involved for computing the Hausdorff distance is $M N$. However, number of points involved for computing HSD is only $2 M$ because only two left and right neighborhoods in 1-D space are required. It is much smaller than $M N$ since always hundreds or thousands points are extracted in an image.

Then, we can analyze the computational complexity of the proposed search strategy as in [16]. Two extreme cases may occur in the search process: first, all $C_{i} \mathrm{~s}$ are replaced by $M_{i}$ s in each stage and we only need to compute HSD value at $M_{i}$ s. If $C_{\text {ave }}$ is the average number of candidates selected for each stage, the number of total search points in this case is

$$
N_{1}=k+1+C_{\text {ave }} \log _{2}\left(\Delta d_{0}\right)
$$

Second, if no $C_{i}$ is replaced by $M_{i}$ in each stage and we have to compute HSD value at $C_{i}+\Delta d$ and $C_{i}-\Delta d$, which results in the number of search points becomes

$$
N_{2}=k+1+2 C_{\text {ave }} \log _{2}\left(\Delta d_{0}\right) .
$$

Here, $\Delta d_{0}$ can be written as

$$
\Delta d_{0}=\frac{L}{k}
$$

Thus, the average number of $N_{1}$ and $N_{2}$ is treated as the final estimation of computational complexity

$$
N_{\text {ave }}=k+1+\frac{3}{2} C_{\text {ave }} \log _{2}\left(\frac{L}{k}\right) \text {. }
$$

\section{Conclusions and Future Work}

In this study, we propose a two-stage matching algorithm for Point Pattern Matching (PPM) using a new ellipse fitting method named Weighted Least Square Fitting (WLSF) and dual Hilbert scans. Compared to the Direct Least Square Fitting (DLSF) method, WLSF shows its robustness to noise and outlier points. The whole algorithm also gives an accurate and fast solution for PPM problem. Future work will aim at solving the weights in WLSF automatically by analyzing the distribution of the point set and extending the algorithm to more real-world applications.

\section{References}

[1] P. Yin, "Particle swarm optimization for point pattern matching," J. Vis. Commun. Image Represent., vol.17, no.12, pp.143-162, 2006.

[2] D.P. Huttenlocher, G.A. Klanderman, and W.J. Rucklidge, "Comparing images using the Hausdorff distance," IEEE Trans. Pattern Anal. Mach. Intell., vol.15, no.12, pp.850-863, 1993.

[3] M.P. Dubuisson and A.K. Jain, "A modified Hausdorff ditance for object matching," Proc. 12th ICPR, pp.566-568, 1994.

[4] A. Agrawal, N. Ansari, and E. Hou, "Evolutionary programming for fast and robust point pattern matching," Proc. IEEE International Conf. on Neural Networks, vol.3, pp.1777-1782, 1994.

[5] S. Umeyama, "Least-squares estimation of transformation parameters between two point patterns," IEEE Trans. Pattern Anal. Mach. Intell., vol.13, no.4, pp.376-380, April 1991.

[6] W.H. Wang and Y.C. Chen, "Point pattern matching by line segments and labels," Electron. Lett., vol.33, no.6, pp.478-479, March 1997.

[7] C. Griffin and P.M. Alexopoulos, "Point pattern matching using centroid bounding," IEEE Trans. Syst. Man Cybern., vol.19, no.5, pp.1274-1276, 1989.

[8] C.F. Olson and D.P. Huttenlocher, "Automatic target recognition by matching oriented edge pixels," IEEE Trans. Image Process., vol.6, no.1, pp.103-113, Jan. 1997.

[9] S. Ranade and A. Rosenfeld, "Point pattern matching by relaxation," Pattern Recognit., vol.12, no.4, pp.269-275, 1980.

[10] H. Ogawa, "Labeled point pattern matching by fuzzy relaxation," Pattern Recognit., vol.17, no.5, pp.569-573, 1984.

[11] D. Mount, N. Netanyahu, and J.L. Moigne, "Efficient algorithms for robust feature matching," Pattern Recognit., vol.32, no.13, pp.1728, 1998.

[12] M. Carcassoni and E. Hancock, "Spectral correspondence for point pattern matching," Pattern Recognit., vol.36, no.1, pp.193-204, Jan. 2003.

[13] L. Zhang, W. Xu, and C. Change, "Genetic algorithm for affine point pattern matching," Pattern Recognit. Lett., vol.24, no.1-3, pp.9-19, 2003.

[14] J. Starink and E. Backer, "Finding point correspondences using simulated annealing," Pattern Recognit., vol.28, no.2, pp.231-240, Feb. 1995.

[15] S. Kamata, M. Niimi, and E. Kawaguchi, "A method of an interactive analysis for multi-dimensional images using a Hilbert curve," IEICE Trans. Inf. \& Syst. (Japanese Edition), vol.J77-D-II, no.7, pp.1255-1264, July 1994.

[16] Y. Wang and H. Kuroda, "Hilbert scanning search algorithm for motion estimation," IEEE Trans. Circuits Syst. Video Technol., vol.9, no.5, pp.683-691, 1999.

[17] B. Moghaddam, K.J. Hintz, and C.V. Stewart, "Space-filling curves for image compression," Proc. SPIE Conf. Automatic Object Recognition, vol.1471, pp.414-421, 1991.

[18] A.C. Ansari and A. Fineberg, "Image data ordering and compression using Peano scan and LOT," IEEE Trans. Consum. Electron., vol.38, no.3, pp.436-445, 1999.

[19] H. Sagan, "A three-dimensional Hilbert-curve," J. Math. Ed. Sc. Tech., vol.24, no.1, pp.541-545, 1993.

[20] J. Zhang, S. Kamata, and Y. Ueshige, "A pseudo-Hilbert scan for arbitrarily-sized arrays," IEICE Trans. Fundamentals, vol.E90-A, no.3, pp.682-690, March 2007.

[21] L. Tian, S. Kamata, K. Tsuneyoshi, and H.J. Tang, "A fast and accurate algorithm for matching images using Hilbert scanning distance with threshold elimination function," IEICE Trans. Inf. \& Syst., vol.E89-D, no.1, pp.290-297, Jan. 2006.

[22] V. Leavers, Shape detection in computer vision using the Hough transform, Springer-Verlag, New York, 1992.

[23] C. Daul, P. Graebling, and E. Hirsch, "From the Hough transform to a new approach for the detection and approximation of elliptical 
arcs," Comput. Vis. Image Understand., vol.72, no.3, pp.215-236, Dec. 1998.

[24] A. Sewisy and F. Leberl, "Detection ellipses by finding lines of symmetry in the images via an hough transform applied to straight lines," Image Vis. Comput., vol.19, no.12, pp.857-866, Oct. 2001.

[25] Z. Liu, H. Qiao, and L. Xu, "Multisets mixture learning-based ellipse detection," Pattern Recognit., vol.39, no.4, pp.731-735, April 2006.

[26] F. Bookstein, "Fitting conic sections to scattered data," Comput. Graph. Image Process., vol.9, no.1, pp.56-71, 1979.

[27] G. Taubin, "Estimation of planar curves, surfaces, and nonplanar space curves defined by implicit equations with applications to edge and range image segmentation," IEEE Trans. Pattern Anal. Mach. Intell., vol.13, no.11, pp.1115-1138, Nov. 1991.

[28] P. Rosin, "A note on the least squares fitting of ellipses," Pattern Recognit. Lett., vol.14, no.10, pp.799-808, 1993.

[29] W. Gander, G. Golub, and R. Strebel, "Least-square fitting of circles and ellipses," BIT, no.43, pp.558-578, 1994.

[30] A. Fitzgibbon, M. Pilu, and R. Fisher, "Direct least square fitting of ellipses," IEEE Trans. Pattern Anal. Mach. Intell., vol.21, no.5, pp.476-480, May 1999.

[31] P. Sampson, "Fitting conic sections to 'very scattered' data: An iterarive refinement of the bookstein algorithm," Comput. Graph. Image Process., vol.18, no.1, pp.97-108, Jan. 1982.

[32] J. Porrill, "Fitting ellipses and predicting confidence envelopes using a bias corrected kalman filter," Image Vis. Comput., vol.8, no.1, pp.37-41, Feb. 1990.

[33] K. Kanatani, "Statistical bias of conic fitting and renormalization," IEEE Trans. Pattern Anal. Mach. Intell., vol.16, no.3, pp.320-326, March 1994.

[34] A. Goshtasby, "Image registration by local approximation methods," Image Vis. Comput., vol.6, no.4, pp.255-261, 1988.

[35] R.I. Hartley and A. Zisserman, Multiple View Geometry in Computer Vision, second ed., Cambridge University Press, ISBN: 0521540518, 2004.

[36] A.K. Jain, L. Hong, and R.M. Bolle, "On-line fingerprint verification,” IEEE Trans. Pattern Anal. Mach. Intell., vol.19, no.4, pp.302314, 1997.

[37] FVC2002 http://bias.cst.unibo.it/fvc2002/

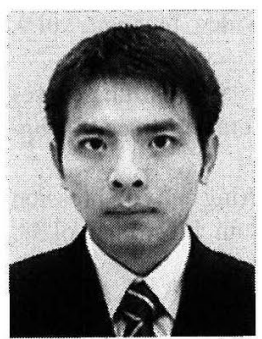

Li Tian is a Ph.D. student of Graduate School of Information, Production and Systems at Waseda University, where he received the M.S. degree in 2006 . He received his bachelor's degree from Xi' an Jiaotong University, China in 2003. His current research interests are in image processing, pattern recognition and computer vision. He is a Research Fellow of the Japan Society for the Promotion of Science, and also a student member of the IEEE.

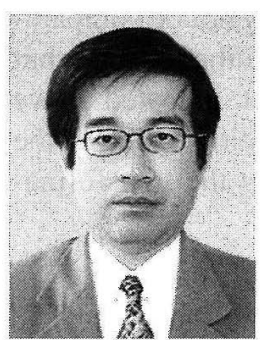

Sei-ichiro Kamata received the M.S. degree in computer science from Kyushu University, Fukuoka, Japan, in 1985, and the Doctor of Engineering degree from the Department of Computer Science, Kyushu Institute of Technology, Kitakyushu, Japan, in 1995. From 1985 to 1988, he was with NEC, Ltd., Kawasaki, Japan. In 1988 , he joined the faculty at Kyushu Institute of Technology. From 1996 to 2003, he was an Associate Professor in the Department of Intelligent Systems, Graduate School of Information Science and Electrical Engineering, Kyushu University. Since 2003, he has been a Professor in Graduate School of Information, Production and Systems, Waseda University. In 1990 and 1994, he was a Visiting Researcher at the University of Maine, Orono. His research interests include image processing, pattern recognition, image compression, and space-filling curve applications. Dr. Kamata is a member of the IEEE. 